\title{
A NOTE ON DISCRETE OPIAL'S INEQUALITY
}

\author{
GOU-SHENG YANG AND CHENG-DAR YOU
}

\section{Introduction}

Z.Opial [2] has proved in 1960 the following result:

Theorem A. If $f^{\prime}$ is a continuous function on $[0, h]$, and if $f(0)=f(h)=0$ and $f(x)>0$ for $x \in(0, h)$, then

$$
\int_{0}^{h}\left|f(x) f^{\prime}(x)\right| d x \leq \frac{h}{4} \int_{0}^{h} f^{\prime}(x)^{2} d x
$$

where the constant $h / 4$ is the best possible.

In 1967 J.S.W.Wong [4] has proved the following discrete analogue of (1).

Theorem B. Let $u_{i}$ be a nondecreasing sequence of nonnegative real numbers, such that $u_{0}=0$. Then, for $p \geq 1$, we have

$$
\sum_{i=1}^{n}\left(u_{i}-u_{i-1}\right) u_{i}^{p} \leq \frac{(n+1)^{p}}{p+1} \sum_{i=1}^{n}\left(u_{i}-u_{i-1}\right)^{p+1} .
$$

Cheng-Ming Lee [1] generalized (2) in the following form:

Theorem C. Let $u_{i}$ be a nondecreasing sequence of nonnegative numbers, such that $u_{0}=0$.

If $p, q>0, p+q \geq 1$ or $p, q<0$, then

$$
\sum_{i=1}^{n}\left(u_{i}-u_{i-1}\right)^{q} u_{i}^{p} \leq K_{n} \sum_{i=1}^{n}\left(u_{i}-u_{i-1}\right)^{p+q}
$$

Received June 25, 1991 
where

$$
K_{0}=\frac{q}{p+q}
$$

and

$$
K_{n}=\max \left(K_{n-1}+\frac{p n^{p-1}}{p+q}, \frac{q(n+1)^{p}}{p+q}\right) \quad(n=1,2, \cdots) .
$$

If $p>0, q<0, p+q \leq 1, p+q \neq 0$ or $p<0, q>0, p+q \geq 1$, then

$$
\sum_{i=1}^{n}\left(u_{i}-u_{i-1}\right)^{q} u_{i}^{p} \geq C_{n} \sum_{i=1}^{n}\left(u_{i}-u_{i-1}\right)^{p+q}
$$

where

$C_{0}=\frac{q}{p+q} \quad$ and $\quad C_{n}=\min \left(C_{n-1}+\frac{p n^{p-1}}{p+q}, \frac{q(n+1)^{p}}{p+q}\right) \quad(n=1,2, \cdots)$. If $p, q \geq 1$, then

$$
K_{n}=\frac{q(n+1)^{p}}{p+q}
$$

If $p \leq 0, q<0$, then

$$
K_{1}=1, \quad \text { and } \quad K_{n}=1+\frac{p}{p+q} \sum_{i=2}^{n} i^{p-1} \quad(n=2,3, \cdots)
$$

If $p \geq 0, p+q<0$, then

$$
C_{1}=1, \quad \text { and } \quad C_{n}^{\prime}=1+\frac{p}{p+q} \sum_{i=2}^{n} i^{p-1} \quad(n=2,3, \cdots) .
$$

Recently, B.G.Pachpatte [3] has proved a new discrete inequality of Opial type involving a function of $n$ independent variables in the following:

Theorem D. If $z$ is a function from $B$ to $R$ such that $\nabla_{1} \cdots \nabla_{n} z(x)$ exist and 


$$
\begin{gathered}
z\left(0, x_{2}, \cdots, x_{n}\right)=z\left(x_{1}, 0, x_{3}, \cdots, x_{n}\right)=\cdots=z\left(x_{1}, \cdots, x_{n-1}, 0\right)=0 . \text { then } \\
\sum_{B}\left|z(x) \| \nabla_{1} \cdots \nabla_{n} z(x)\right| \leq\left(\sum_{B}\left(\prod_{i=1}^{n} x_{i}\right)\left(\sum_{B_{x}}\left|\nabla_{1} \cdots \nabla_{n} z(y)\right|^{2}\right)\right)^{\frac{1}{2}} \\
\bullet\left(\sum_{B}\left|\nabla_{1} \cdots \nabla_{n} z(x)\right|^{2}\right)^{\frac{1}{2}},
\end{gathered}
$$

where

$$
N_{0}=\{0,1,2, \cdots\}, \quad B=\prod_{i=1}^{n}\left[0, C_{i}\right] \subset N_{0}^{n},
$$

and $\nabla_{1} z\left(x_{1}, x_{2}, \cdots, x_{n}\right)=z\left(x_{1}, x_{2}, \cdots, x_{n}\right)-z\left(x_{1}-1, x_{2}, \cdots, x_{n}\right)$,

$$
\begin{gathered}
\nabla_{n} z\left(x_{1}, x_{2}, \cdots, x_{n}\right)=z\left(x_{1}, \cdots, x_{n-1}, x_{n}\right)-z\left(x_{1}, \cdots, x_{n-1}, x_{n}-1\right), \\
\nabla_{1} \nabla_{2} z\left(x_{1}, x_{2}, \cdots, x_{n}\right)=\nabla_{1}\left[z\left(x_{1}, x_{2}, \cdots, x_{n}\right)-z\left(x_{1}, x_{2}-1, x_{3}, \cdots, x_{n}\right)\right] \\
\sum_{B} z(x)=\sum_{x_{1}=1}^{c_{1}} \cdots \sum_{x_{n}=1}^{C_{n}} z\left(x_{1}, x_{2}, \cdots, x_{n}\right), \\
\sum_{B_{x}} z(y)=\sum_{y_{1}=1}^{x_{1}} \cdots \sum_{y_{n}=1}^{x_{n}} z\left(y_{1}, y_{2}, \cdots, y_{n}\right) .
\end{gathered}
$$

The purpose of the present note is to generalize the inequality (2) as well as the inequality (5).

\section{Discrete Opial's inequalities in one-dimention}

Lemma 1. Let $u_{0}, u_{1}, \cdots u_{n}$ be a sequence of real numbers, such that $u_{0}=0$. If $p, q \geq 1$, then

$$
\sum_{i=1}^{n}\left|u_{i}-u_{i-1}\right|^{q}\left|u_{i}\right|^{p} \leq \frac{q(n+1)^{p}}{p+q} \sum_{i=1}^{n}\left|u_{i}-u_{i-1}\right|^{p+q}
$$


Proof. Let $x_{i}=\left|u_{i}-u_{i-1}\right|$. Then $\left|u_{i}\right| \leq \sum_{j=1}^{i} x_{j}$, so that.

$$
\sum_{i=1}^{n}\left|u_{i}-u_{i-1}\right|^{q}\left|u_{i}\right|^{p} \leq \sum_{i=1}^{n} x_{i}^{q}\left(\sum_{j=1}^{i} x_{j}\right)^{p} .
$$

It suffices to prove

$$
\sum_{i=1}^{n} x_{i}^{q}\left(\sum_{j=1}^{i} x_{j}\right)^{p} \leq \frac{q(n+1)^{p}}{p+q} \sum_{i=1}^{n} x_{i}^{p+q}
$$

We will prove (8) by induction.

Since $2^{p} \geq p+1$ for $p \geq 1$, it follows that

$$
x_{1}^{p+q} \leq \frac{q \cdot 2^{p}}{p+q} x_{1}^{p+q} .
$$

This shows that ( 8 ) holds if $n=1$. Assume (8) holds for $n=k$, and observe

$$
\begin{aligned}
\sum_{i=1}^{k+1} x_{i}^{q}\left(\sum_{j=1}^{i} x_{j}\right)^{p} & =\sum_{i=1}^{k} x_{i}^{q}\left(\sum_{j=1}^{i} x_{j}\right)^{p}+x_{k+1}^{q}\left(\sum_{j=1}^{k+1} x_{j}\right)^{p} \\
& \leq \frac{q(k+1)^{p}}{p+q} \sum_{i=1}^{k} x_{i}^{p+q}+x_{k+1}^{q}\left(\sum_{j=1}^{k+1} x_{j}\right)^{p} \\
& =\frac{q(k+1)^{p}}{p+q}\left(\sum_{i=1}^{k} x_{i}^{p+q}+\frac{p+q}{q} x_{k+1}^{q} y_{k+1}^{p}\right)
\end{aligned}
$$

where $y_{n}=\frac{1}{n} \sum_{i=1}^{n} x_{i}$.

By Young's inequality we have

$$
\begin{aligned}
\frac{p+q}{q} x_{k+1}^{q} y_{k+1}^{p} & \leq \frac{p+q}{q}\left(\frac{q}{p+q} x_{k+1}^{\frac{p+q}{q}}+\frac{p}{p+q} y_{k+1}^{p \frac{p+q}{p}}\right) \\
& =x_{k+1}^{p+q}+\frac{p}{q} y_{k+1}^{p+q} .
\end{aligned}
$$

Using II ölder's inequality, we may show that

$$
y_{k+1}^{p+q} \leq \frac{1}{k+1} \sum_{i=1}^{k+1} x_{i}^{p+q}
$$


Also we have $(k+2)^{p}-(k+1)^{p} \geq p(k+1)^{p-1} \geq \underset{q}{p}(k+1)^{p-1}$.

Substituting these estimates into (9), we find

$$
\begin{aligned}
\sum_{i=1}^{k+1} x_{i}^{q}\left(\sum_{j=1}^{i} x_{j}\right)^{p} & \leq \frac{q(k+1)^{p}}{p+q}\left(\sum_{i=1}^{k+1} x_{i}^{p+q}+\frac{p}{q} \cdot \frac{1}{k+1} \sum_{i=1}^{k+1} x_{i}^{p+q}\right) \\
& \leq \frac{q(k+2)^{p}}{p+q} \sum_{i=1}^{k+1} x_{i}^{p+q} .
\end{aligned}
$$

This completes the proof.

Lemma 2. Let $u_{0}, u_{1}, u_{2}, \cdots, u_{N}$ be any sequence of real numbers, such that $u_{N}=0$. If $p, q \geq 1$, then

$$
\sum_{i=n}^{N-1}\left|u_{i}-u_{i-1}\right|^{q}\left|u_{i}\right|^{p} \leq \frac{q(N-n+1)^{p}}{p+q} \sum_{i=n}^{N}\left|u_{i}-u_{i-1}\right|^{p+q} .
$$

Proof.

Let $x_{i}=\left|u_{i}-u_{i-1}\right|$.Then $\left|u_{i}\right| \leq \sum_{j=i+1}^{N} x_{j}$, so that

$$
\sum_{i=n}^{N-1}\left|u_{i}-u_{i-1}\right|^{q}\left|u_{i}\right|^{p} \leq \sum_{i=n}^{N-1} x_{i}^{q}\left(\sum_{j=i+1}^{N} x_{j}\right)^{p}
$$

We will show by induction that

$$
\sum_{i=n}^{N-1} x_{i}^{q}\left(\sum_{j=i+1}^{N} x_{j}\right)^{p} \leq \frac{q(N-n+1)^{p}}{p+q} \sum_{i=n}^{N} x_{i}^{p+q}
$$

By Young's inequality, we have

$$
\begin{aligned}
x_{N-1}^{q} x_{N}^{p} & \leq \frac{q}{p+q} x_{N-1}^{p+q}+\frac{p}{p+q} x_{N}^{p+q} \\
& \leq \frac{q \cdot 2^{p}}{p+q} \sum_{i=N-1}^{N} x_{i}^{p+q}
\end{aligned}
$$

so that (11) holds if $n=N-1$. Assume (11) holds for $n=k$, where $k<N-1$. 
Since

$$
\begin{aligned}
\sum_{i=k-1}^{N-1} x_{i}^{q}\left(\sum_{j=i+1}^{N} x_{j}\right)^{p} & =\sum_{i=k}^{N-1} x_{i}^{q}\left(\sum_{j=i+1}^{N} x_{j}\right)^{p}+x_{k-1}^{q}\left(\sum_{j=k}^{N} x_{j}\right)^{p} \\
& \leq \frac{q(N-k+1)^{p}}{p+q} \sum_{i=k}^{N} x_{i}^{p+q}+x_{k-1}^{q}\left(\sum_{j=k}^{N} x_{j}\right)^{p} .
\end{aligned}
$$

Let

$$
y_{k}=\frac{1}{N-k+1}\left(\sum_{j=k}^{N} x_{j}\right)
$$

Then

$$
x_{k-1}^{q}\left(\sum_{j=k}^{N} x_{j}\right)^{p}=(N-k+1)^{p} x_{k-1}^{q} y_{k}^{p} .
$$

It follows from Young's inequality that

$$
(N-k+1)^{p} x_{k-1}^{q} y_{k}^{p} \leq(N-k+1)^{p}\left(\frac{q}{p+q} x_{k-1}^{p+q}+\frac{p}{p+q} y_{k}^{p+q}\right) .
$$

Using Hölder's inequality, we have

$$
y_{k}^{p+q}=\left(\frac{1}{N-k+1}\right)^{p+q}\left(\sum_{j=k}^{N} x_{j}\right)^{p+q} \leq \frac{1}{N-k+1} \sum_{j=k}^{N} x_{j}^{p+q}
$$

Substituting these estimates into (12), we find

$$
\begin{aligned}
& \sum_{i=k-1}^{N-1} x_{i}^{q}\left(\sum_{j=i+1}^{N} x_{j}\right)^{p} \\
& \quad \leq \frac{q(N-k+1)^{p}}{p+q} \sum_{i=k-1}^{N} x_{i}^{p+q}+\frac{p(N-k+1)^{p-1}}{p+q} \sum_{j=k}^{N} x_{j}^{p+q} \\
& \quad \leq\left(\frac{q(N-k+1)^{p}}{p+q}+\frac{p(N-k+1)^{p-1}}{p+q}\right) \sum_{i=k-1}^{N} x_{i}^{p+q} \\
& \quad \leq \frac{q(N-k+2)^{p}}{p+q} \sum_{i=k-1}^{N} x_{i}^{p+q} .
\end{aligned}
$$


This completes the proof.

Theorem 1. Let $u_{0}, u_{1}, u_{2}, \cdots, u_{N} \in R$ be a sequence of real numbers such that $u_{0}=u_{N}=0$. Then for $p, q \geq 1$, we have

$$
\sum_{i=1}^{N-1}\left|u_{i}-u_{i-1}\right|^{q}\left|u_{i}\right|^{p} \leq \frac{q(N+1)^{p}}{2^{p}(p+q)} \sum_{i=1}^{N}\left|u_{i}-u_{i-1}\right|^{p+q}
$$

where $N$ is odd, and

$$
\sum_{i=1}^{N-1}\left|u_{i}-u_{i-1}\right|^{q}\left|u_{i}\right|^{p} \leq \frac{q(N+2)^{p}}{2^{p}(p+q)} \sum_{i=1}^{N}\left|u_{i}-u_{i-1}\right|^{p+q}
$$

where $N$ is even.

Proof. $1^{\circ} N$ is odd:

If $N=1$, then there is nothing to prove, so assume $N>1$.

Let $n=\frac{N-1}{2}$. By Iemma 1, we have

$$
\begin{aligned}
\sum_{i=1}^{n}\left|u_{i}-u_{i-1}\right|^{q}\left|u_{i}\right|^{p} & \leq \frac{q(n+1)^{p}}{p+q} \sum_{i=1}^{n}\left|u_{i}-u_{i-1}\right|^{p+q} \\
& =\frac{q\left(\frac{N-1}{2}+1\right)^{p}}{p+q} \sum_{i=1}^{\frac{N-1}{2}}\left|u_{i}-u_{i-1}\right|^{p+q}
\end{aligned}
$$

and by Lemma 2, we have

$$
\begin{aligned}
\sum_{i=n+1}^{N-1}\left|u_{i}-u_{i-1}\right|^{q}\left|u_{i}\right|^{p} & \leq \frac{q(N-n)^{p}}{p+q} \sum_{i=n+1}^{N}\left|u_{i}-u_{i-1}\right|^{p+q} \\
& =\frac{q\left(N-\frac{N-1}{2}\right)^{p}}{p+q} \sum_{i=\frac{N+1}{2}}^{N}\left|u_{i}-u_{i-1}\right|^{p+q} .
\end{aligned}
$$

Adding (15) and (16) to infer that

$$
\sum_{i=1}^{N-1}\left|u_{i}-u_{i-1}\right|^{q}\left|u_{i}\right|^{p} \leq \frac{q(N+1)^{p}}{2^{p}(p+q)} \sum_{i=1}^{N}\left|u_{i}-u_{i-1}\right|^{p+q} .
$$


$2^{\circ} \mathrm{N}$ is even: Let $u_{N+1}=0$. Then it follows from (13) that

$$
\sum_{i=1}^{N}\left|u_{i}-u_{i-1}\right|^{q}\left|u_{i}\right|^{p} \leq \frac{q(N+2)^{p}}{2^{p}(p+q)} \sum_{i=1}^{N+1}\left|u_{i}-u_{i-1}\right|^{p+q} .
$$

Hence

$$
\sum_{i=1}^{N-1}\left|u_{i}-u_{i-1}\right|^{q}\left|u_{i}\right|^{p} \leq \frac{q(N+2)^{p}}{2^{p}(p+q)} \sum_{i=1}^{N}\left|u_{i}-u_{i-1}\right|^{p+q} .
$$

This completes the proof.

\section{Discrete Opial's inequality in n-dimensions}

Lemma 3. If $z$ is a function from $B$ to $R$ such that $\nabla_{1} \cdots \nabla_{n} z(x)$ exist and $z\left(0, x_{2}, \cdots, x_{n}\right)=z\left(x_{1}, 0, x_{3}, \cdots, x_{n}\right)=\cdots=z\left(x_{1}, \cdots, x_{n-1}, 0\right)=0$. then

$$
\begin{aligned}
\sum_{B}|z(x)|^{p}\left|\nabla_{1} \cdots \nabla_{n} z(x)\right|^{q} \leq & \left(\sum_{B}\left(\prod_{i=1}^{n} x_{i}\right)^{p+q-1}\right)^{\frac{p}{p+q}} \\
& \left(\sum_{B}\left|\nabla_{1} \cdots \nabla_{n} z(x)\right|^{p+q}\right) .
\end{aligned}
$$

for $p+q>1$, and $p, q>0$. The notations and definitions are the same as in Theorem $D$.

Proof. We have

$$
z(x)=\sum_{B_{x}} \nabla_{1} \cdots \nabla_{n} z(y)
$$

Using Hölder's inequality, we have

$$
\begin{aligned}
|z(x)| & \leq \sum_{B_{x}}\left|\nabla_{1} \cdots \nabla_{n} z(y)\right| \\
\leq & \left(\sum_{B_{x}} 1^{\frac{p+q}{p+q-1}}\right)^{\frac{p+q-1}{p+q}} \\
& \cdot\left(\sum_{B_{x}}\left|\nabla_{1} \cdots \nabla_{n} z(y)\right|^{p+q}\right)^{\frac{1}{p+q}},
\end{aligned}
$$


so that

$$
|z(x)|^{p+q} \leq\left(\prod_{i=1}^{n} x_{i}\right)^{p+q-1}\left(\sum_{B_{x}}\left|\nabla_{1} \cdots \nabla_{n} z(y)\right|^{p+q}\right)
$$

Using Hölder's inequality again we have

$$
\begin{aligned}
\sum_{B}|z(x)|^{p}\left|\nabla_{1} \cdots \nabla_{n} z(x)\right|^{q} \leq\left(\sum_{B}|z(x)|^{p+q}\right)^{\frac{p}{p+q}} \\
\bullet\left(\sum_{B}\left|\nabla_{1} \cdots \nabla_{n} z(x)\right|^{p+q}\right)^{\frac{q}{p+q}}
\end{aligned}
$$

It follows from (18) that

$$
\begin{aligned}
& \sum_{B}|z(x)|^{p}\left|\nabla_{1} \cdots \nabla_{n} z(x)\right|^{q} \\
\leq & \left(\sum_{B}\left(\prod_{i=1}^{n} x_{i}\right)^{p+q-1}\left(\sum_{B_{x}}\left|\nabla_{1} \cdots \nabla_{n} z(y)\right|^{p+q}\right)\right)^{\frac{p}{p+q}} \\
& \cdot\left(\sum_{B}\left|\nabla_{1} \cdots \nabla_{n} z(x)\right|^{p+q}\right)^{\frac{q}{p+q}} \\
\leq & \left(\sum_{B}\left(\prod_{i=1}^{n} x_{i}\right)^{p+q-1} \cdot \sum_{B}\left|\nabla_{1} \cdots \nabla_{n} z(x)\right|^{p+q}\right)^{\frac{p}{p+q}} \\
& \cdot\left(\sum_{B}\left|\nabla_{1} \cdots \nabla_{n} z(x)\right|^{p+q}\right)^{\frac{q}{p+q}} \\
= & \left(\sum_{B}\left(\prod_{i=1}^{n} x_{i}\right)^{p+q-1}\right)^{\frac{p}{p+q}} \\
0 & \left(\sum_{B}\left|\nabla_{1} \cdots \nabla_{n} z(x)\right|^{p+q}\right)^{p}
\end{aligned}
$$

This completes the proof.

In case $n=2$, and $B=\left[0, m_{1}\right] \times\left[0, m_{2}\right]$ we have

Lemma 4. If $B_{1}=\left[0, c_{1}\right] \times\left[0, c_{2}\right], z$ is a function from $B_{1}$ to $R$ such that $\nabla_{1} \nabla_{2} z(x)$ exists and $z\left(0, x_{2}\right)=z\left(x_{1}, 0\right)=0$ for $x_{1} \in\left\{0,1, \cdots c_{1}\right\}, x_{2} \in$ $\left\{0,1, \cdots c_{2}\right\}$, then 


$$
\begin{aligned}
\sum_{B_{1}}|z(x)|^{p}\left|\nabla_{1} \nabla_{2} z(x)\right|^{q} \leq & \left(\sum_{B_{1}}\left(\prod_{i=1}^{2} x_{i}\right)^{p+q-1}\right)^{\frac{p}{p+q}} \\
& \bullet\left(\sum_{B_{1}}\left|\nabla_{1} \nabla_{2} z(x)\right|^{p+q}\right) .
\end{aligned}
$$

Proof. This follows from Lemma 3.

Lemma 5. If $B_{2}=\left[0, c_{1}\right] \times\left[c_{2}+1, m_{2}\right], z$ is a function from $B_{2}$ to $R$ such that $\nabla_{1} \nabla_{2} z(x)$ exists and $z\left(0, x_{2}\right)=z\left(x_{1}, m_{2}\right)=0$ for $x_{1} \in\left\{0,1, \cdots c_{1}\right\}, x_{2} \in$ $\left\{c_{2}+1, c_{2}+2, \cdots m_{2}\right\}$, then

$$
\begin{aligned}
\sum_{B_{2}}|z(x)|^{p}\left|\nabla_{1} \nabla_{2} z(x)\right|^{q} \leq\left(\sum_{B_{2}}\left(x_{1}\left(m_{2}-x_{2}\right)\right)^{p+q-1}\right)^{\frac{p}{p+q}} \\
\circ\left(\sum_{B_{2}}\left|\nabla_{1} \nabla_{2} z(x)\right|^{p+q}\right) .
\end{aligned}
$$

Proof. we have

$$
-z(x)=\sum_{y_{1}=1}^{x_{1}} \sum_{y_{2}=x_{2}+1}^{m_{2}} \nabla_{1} \nabla_{2} z(y)
$$

and the rest of the proof is similar to Lemma. 3 .

Lemma 6. If $B_{3}=\left[c_{1}+1, m_{1}\right] \times\left[0, c_{2}\right], z$ is a function from $B_{3}$ to $R$ such that $\nabla_{1} \nabla_{2} z(x)$ exists and $z\left(m_{1}, x_{2}\right)=z\left(x_{1}, 0\right)=0$ for $x_{1} \in\left\{c_{1}+1, c_{1}+\right.$ $\left.2, \cdots m_{1}\right\}, x_{2} \in\left\{0,1, \cdots c_{2}\right\}$, then

$$
\begin{aligned}
\sum_{B_{3}}|z(x)|^{p}\left|\nabla_{1} \nabla_{2} z(x)\right|^{q} \leq & \left(\sum_{B_{3}}\left(\left(m_{1}-x_{1}\right) x_{2}\right)^{p+q-1}\right)^{\frac{p}{p+q}} \\
& \circ\left(\sum_{B_{3}}\left|\nabla_{1} \nabla_{2} z(x)\right|^{p+q}\right) .
\end{aligned}
$$


Proof. we have

$$
-z(x)=\sum_{y_{1}=x_{1}+1}^{m_{1}} \sum_{y_{2}=1}^{x_{2}} \nabla_{1} \nabla_{2} z(y)
$$

and the rest of the proof is similar to Lemma 3 .

Lemma 7. If $B_{4}=\left[c_{1}+1, m_{1}\right] \times\left[c_{2}+1, m_{2}\right], z$ is a function from $B_{4}$ to $R$ such that $\nabla_{1} \nabla_{2} z(x)$ exists and $z\left(m_{1}, x_{2}\right)=z\left(x_{1}, m_{2}\right)=0$ for $x_{1} \in\left\{c_{1}+1, c_{1}+\right.$ $\left.2, \cdots m_{1}\right\}, x_{2} \in\left\{c_{2}+1, c_{2}+2, \cdots m_{2}\right\}$, then

$$
\begin{gathered}
\sum_{B_{4}}|z(x)|^{p}\left|\nabla_{1} \nabla_{2} z(x)\right|^{q} \leq\left(\sum_{B_{4}}\left(\left(m_{1}-x_{1}\right)\left(m_{2}-x_{2}\right)\right)^{p+q-1}\right)^{\frac{p}{p+q}} \\
\circ\left(\sum_{B_{4}}\left|\nabla_{1} \nabla_{2} z(x)\right|^{p+q}\right) .
\end{gathered}
$$

Proof. we have

$$
z(x)=\sum_{y_{1}=x_{1}+1}^{m_{1}} \sum_{y_{2}=x_{2}+1}^{m_{2}} \nabla_{1} \nabla_{2} z(y)
$$

and the rest of the proof is similar to Lemma 3 .

Combining Lemma 4,Lemma 5,Lemma 6 and Lemma 7, we have

Theorem 2. If $B=\left[0, m_{1}\right] \times\left[0, m_{2}\right], m_{i}$ is odd for $i=1,2$. $z$ is a function from $B$ to $R$ such that $\nabla_{1} \nabla_{2} z(x)$ exists and $z\left(0, x_{2}\right)=z\left(x_{1}, 0\right)=z\left(m_{1}, x_{2}\right)=$ $z\left(x_{1}, m_{2}\right)=0$ for $x_{1} \in\left\{0,1, \cdots m_{1}\right\}, x_{2} \in\left\{0,1, \cdots m_{2}\right\}$, then

$$
\begin{gathered}
\sum_{B}|z(x)|^{p}\left|\nabla_{1} \nabla_{2} z(x)\right|^{q} \leq \prod_{i=1}^{2}\left(1+2^{p+q-1}+\cdots+\left(\frac{m_{i}-1}{2}\right)^{p+q-1}\right)^{\frac{p}{p+q}} \\
\circ\left(\sum_{B}\left|\nabla_{1} \nabla_{2} z(x)\right|^{p+q}\right) .
\end{gathered}
$$




\section{Proof.}

Let $c_{i}=\frac{m_{i}-1}{2} \quad i=1,2$. Then by (19), (20),(21) and (22) we have

$$
\begin{aligned}
\sum_{B_{m}}|z(x)|^{p}\left|\nabla_{1} \nabla_{i} z(x)\right|^{q} \leq \prod_{i=1}^{2} & \left(1+2^{p+q-1}+\cdots+\left(\frac{m_{i}-1}{2}\right)^{p+q-1}\right)^{\frac{p}{p+q}} \\
& \cdot\left(\sum_{B_{m}}\left|\nabla_{1} \nabla_{2} z(x)\right|^{p+q}\right),(m=1,2,3,4) .
\end{aligned}
$$

Therefore

$$
\begin{aligned}
& \sum_{B}|z(x)|^{p}\left|\nabla_{1} \nabla_{2} z(x)\right|^{q}= \sum_{m=1}^{4} \sum_{B_{m}}|z(x)|^{p}\left|\nabla_{1} \nabla_{2} z(x)\right|^{q} \\
& \leq \sum_{m=1}^{4}\left(\prod_{i=1}^{2}\left(1+2^{p+q-1}+\cdots+\left(\frac{m_{i}-1}{2}\right)^{p+q-1}\right)^{\frac{p}{p+q}}\right. \\
&\left.\quad \sum_{B_{m}}\left|\nabla_{1} \nabla_{2} z(x)\right|^{p+q}\right) . \\
&=\prod_{i=1}^{2}\left(1+2^{p+q-1}+\cdots+\left(\frac{m_{i}-1}{2}\right)^{p+q-1}\right)^{\frac{p}{p+q}} \\
& \quad\left(\sum_{B}\left|\nabla_{1} \nabla_{2} z(x)\right|^{p+q}\right) .
\end{aligned}
$$

This completes the proof.

\section{References}

[1] Lee, Cheng-Ming, "On a discrete analogue of inequalities of Opial and Yang," Canad. Math. Bull. 11, 73-77, 1968.

[2] Opial, Z., "Sur une inégalité.," Ann. Polon. Math. 8, 29-32, 1960.

[3] Pachpatte, B.G., "Opial type inequality in several variables," Tamkang J. Math. Vol 22. No.1, 7-11, 1991.

[4] Wong, J.S.W., "A discrete analogue of Opial's inequality," Canad. Math. Bull. 10, 115$118,1967$. 